\title{
Clinical characteristics and precipitating factor(s) associated with diabetic ketoacidosis presentation in children with newly diagnosed diabetes
}

\section{ABSTRACT}

Background. To compare the demographic and clinical characteristics of children with newly clinically diagnosed type 1 diabetes (T1DM) who presented with diabetic ketoacidosis (DKA) versus non DKA presentation and to identify the precipitating factor(s) related to progression to DKA.

Methods. Over a 3 month period, 99 patients newly diagnosed with T1DM were recruited from Diabetes, Endocrine and Metabolism Pediatric Unit (DEMPU), Cairo University, with 53 patients presented with DKA and 46 were non DKA.

Results. Polyuria, polydipsia, weight loss, polyphagia and nocturia were the most common symptoms preceding the diagnosis among the whole study group (93.8\%, 92\% and $80.8 \%, 76.8 \%, 46.5 \%$ respectively) with no difference between DKA and non DKA groups. Delayed diagnosis occurred in $98.1 \%$ and $58.7 \%$ of DKA and non DKA groups respectively. In the DKA group the diagnosis of diabetes was missed in $69.8 \%$ and in $28.3 \%$ the initiation of insulin therapy was delayed despite diagnosis.

Multivariate analysis performed to identify the most significant precipitating factor(s) associated with the development of DKA at diabetes diagnosis showed

Address for correspondence:

Noha Arafa

Cairo University, Kasr El Aini

Faculty of medicine, Pediatric Department

35221 Elsaraya Street, Cairo, Egypt

e-mail: Noha.hussein@kasralainy.edu.eg

Clinical Diabetology 2020, 9; 5: 286-292

DOI: $10.5603 /$ DK.2020.0042

Received: 12.05.2020

Accepted: 03.08.2020 that delayed start of insulin therapy was the most significant factor $(O R=1.267, P$ value $=0.023)$. Conclusion. The prevalence of DKA is high among Egyptian children at diagnosis of type 1 diabetes. It is not only caused by misdiagnosis and mismanagement of diabetes, but also delayed initiation of insulin therapy in those diagnosed. This highlights the importance of increasing awareness concerning clinical features of diabetes in children and the urgency of insulin therapy among primary health care professionals and the community. (Clin Diabet 2020; 9; 5: 286-292)

Key words: diabetic ketoacidosis, clinical characteristics, precipitating factors, children

\section{Introduction}

The epidemiologic features of type 1 diabetes mellitus (T1DM) and thus DKA are continuously changing. The worldwide incidence of the disease has increased, the reasons for this are yet unknown. Non genetic, environmental causes are incriminated in the rapid rise of the disease incidence [1]. Egypt is one of the countries of the Middle East and North Africa (MENA) region. According to International Diabetes Federation (IDF) report of 2019, the number of children and adolescents (less than 19 years old) with type 1 diabetes was 149,400 cases in MENA region. The number of newly diagnosed patients was 20,800 annually according to the same report [2]. In Egypt, the prevalence of type 1 diabetes estimates in 1992 was 8.0 cases/100,000 [3]. Another study in 2005 revealed the overall incidence was 3.5/100,000 and the estimated prevalence of type 1 diabetes was 38/100,000 [4]. A more recent 
epidemiological study for prevalence of type 1 diabetes in Egyptian subpopulation living in Nile Delta (North Egypt) between 1994 and 2011 was 1.9/100,000 in 1994 reised to $26.8 / 100000$ in 2011 [5]. The incidence and prevalence of type 1 diabetes in Egypt must have been considerably changed although there is lack of nationwide diabetes registry.

Diabetic ketoacidosis (DKA) is an acute life threatening complication of type 1 diabetes and fundamental cause of morbidity and mortality among children with type 1 diabetes as well as s extra healthcare expenses $[6,7]$.

Most of DKA episodes are theoretically preventable through early diagnosis of diabetes and proper insulin management [1], yet to date, DKA frequency at diagnosis of type 1 diabetes in children is still undetermined. Previous report from 24 centers in Europe collected as part of the EURODIAB revealed variable frequency of DKA at diagnosis of type 1 diabetes ranged from $11 \%$ to $67 \%$ [8]. Incidence and rate of complications of DKA and its treatment are largely dependent on various parameters including T1DM incidence in a certain area, age of the child, and access to healthcare facilities for the patients and their families [9].

Previous reports from Egypt regarding frequency of DKA at diagnosis of type 1 diabetes revealed variable rates ranging from 18.6 to $80 \%$, most of these studies are retrospective and cross sectional single center experiences [10-12]. Another recent study recorded DKA as the first presentation in $71 \%$ of toddler at the initial diagnosis of type 1 diabetes [13]. The development of DKA at the diagnosis of type 1 diabetes is associated with poor outcome in the form of more future psychological disorders, less rates of partial remission, and excess healthcare expenses [6]. More understanding of the frequency and the risk factors related to the development of DKA at the time of T1DM diagnosis in children can provide a guide for proper interventional steps for decreasing DKA episodes and consequently healthcare improvement for better patients outcome and lower healthcare expenditures. Thus we aimed to describe and compare the demographic and clinical characteristics of children with newly diagnosed type 1 diabetes who presented with DKA versus those who presented with hyperglycemia (non DKA) and to identify the precipitating factor(s) related to progression to DKA.

\section{Methods}

This is a prospective study was conducted over 3 months period from May to August 2016 at the Diabetes Endocrine and Metabolism Pediatric Unit (DEMPU) at the Children's hospital of Cairo University. The study included all children younger than 15 years of age who were presenting with newly clinically diagnosed type 1 diabetes, and were admitted for management during the study period either in the DEMPU ICU if ketoacidosis or in the inpatient ward if non DKA (hyperglycemia with or without ketonuria). The diagnosis of T1D and DKA were made according to the International Society of Pediatric and Adolescent Diabetes consensus guidelines [14]. After consent, parents were asked to provide more detailed information than that is regularly requested on admission records at DEMPU.

Requested information was collected from all the patients' parents/guardians by the same investigator, and included: chronological age, residence area, number of siblings and order of child within siblings, family history of diabetes including age at onset and mode of therapy in $1^{\text {st }}, 2^{\text {nd }}$ and $3^{\text {rd }}$ degree relatives, availability of previous knowledge about diabetes, preceding symptoms and their duration before diagnosis of diabetes, possible precipitating factors, history of any misdiagnoses, timing of the start of insulin therapy and history of any mismanagement after being diagnosed with diabetes (diet planning alone, exercise with diet panning, oral hypoglycemic drugs etc.), and availability of health insurance coverage.

The study protocol was approved by the local research ethics committee of the pediatric department at Cairo University.

\section{Statistical methods}

The data were processed and analyzed with SPSS 15.0 statistical package software (SPSS Inc., Chicago, Illinois, USA). Qualitative variables were shown as numbers and percentages and quantitative variables were demonstrated as means \pm SD. The chi-square test and Fisher's exact test were used to compare the qualitative data. The differences between quantitative groups with normal distribution were evaluated with student's t-test or the analysis of variance. The differences between quantitative groups with abnormal distribution were evaluated with either Mann-Whitney $U$ test or Kruskal-Wallis test. A P value of $\leq 0.05$ was considered as statistically significant. Multivariate analysis was done to predict the most significant risk factor(s) associated with the development of DKA at the time of diabetes diagnosis.

\section{Results}

Over the 3 months study period, a total of 99 patients with newly diagnosed type 1 diabetes were included; 53 (24 females and 29 males) presented with DKA with variable severity and 46 (23 females and 23 males) with non DKA presentation. Twenty-six patients 
Table 1. Comparison of the demographic data of the two studied groups (DKA and non DKA), total $=99$ patients with newly diagnosed type 1 diabetes

\begin{tabular}{|c|c|c|c|}
\hline & $\begin{array}{c}\text { DKA group }(n=53) \\
(53.5 \%)\end{array}$ & $\begin{array}{c}\text { Non DKA group }(n=46) \\
(46.5 \%)\end{array}$ & $P$ value \\
\hline \multicolumn{4}{|l|}{ Sex } \\
\hline Female & $24(45.3 \%)$ & $23(50 \%)$ & 0.659 \\
\hline Male & $29(54.7 \%)$ & $23(50 \%)$ & \\
\hline \multicolumn{4}{|l|}{ Chronological age (years) } \\
\hline Mean \pm SD (range) & $6.89 \pm 3.63(1.0-14.0)$ & $6.75 \pm 3.54(2.0-13.0)$ & $>0.05$ \\
\hline \multicolumn{4}{|l|}{ Residence } \\
\hline Rural & $11(20.8 \%)$ & $8(17.4 \%)$ & 0.835 \\
\hline Urban & $10(18.9 \%)$ & $11(23.9 \%)$ & \\
\hline Slum & $32(60.4 \%)$ & $27(58.7 \%)$ & \\
\hline Positive parental consanguinity & $12(22.6 \%)$ & $13(28.3 \% 0$ & 0.634 \\
\hline \multicolumn{4}{|l|}{ Order of child in the family } \\
\hline $1^{\text {st }}$ & $16(30.2 \%)$ & $18(39.1 \%)$ & 0.335 \\
\hline $2^{\text {nd }}$ & $17(32.1 \%)$ & $17(37 \%)$ & \\
\hline$\geq 3^{\text {rd }}$ & $20(37.7 \%)$ & $11(23.9 \%)$ & \\
\hline \multicolumn{4}{|l|}{ Number of siblings in family } \\
\hline 1 & $2(3.8 \%)$ & $3(6.5 \%)$ & 0.078 \\
\hline 2 & $11(20.8 \%)$ & $9(19.6 \%)$ & \\
\hline$\geq 3$ & $40(75.4 \%)$ & $34(73.9 \%)$ & \\
\hline Positive family history of diabetes & $30(56.6 \%)$ & $24(52.2 \%)$ & 0.489 \\
\hline Health insurance Availability & $29(54.7 \%)$ & $24(52.2 \%)$ & 0.814 \\
\hline Previous knowledge about diabetes & $14(26.4 \%)$ & $15(32.6 \%)$ & 0.092 \\
\hline \multicolumn{4}{|c|}{ First degree family member with diabetes } \\
\hline Yes & $23(43.4 \%)$ & $8(17.4 \%)$ & 0.005 \\
\hline No & $30(56.6 \%)$ & $38(82.6 \%)$ & \\
\hline
\end{tabular}

DKA — diabetic ketoacidosis

presented with severe DKA (representing $46 \%$ of the DKA group and $26 \%$ of the total number of patients as a whole). Only 9 patients ( $9 \%$ ) of the whole study group presented with hyperglycemia without ketonuria or ketosis.

Positive parental consanguinity was found in 25 (25.3\%) patients among the whole studied groups. Positive family history of diabetes was detected in 54 (54.5\%) patients among the whole recruited groups. There was significantly higher number of patients with first degree relative with diabetes among DKA group $(P$ value $=0.005)$. Comparison between DKA and non DKA groups revealed no significant difference as regards sex, mean ( \pm SD) chronological age at presentation, residence area, parental consanguinity, birth order in the family, number of siblings in the family, family history of diabetes, type of diabetes in the family, and availability of health insurance coverage. As regards previous knowledge about diabetes, the majority of the patients' guardians in both groups had no clear knowledge about diabetes (symptoms and management), with no statistically significant difference between the two groups, Table 1.

The most common symptoms preceding the diagnosis were polyuria, polydipsia, weight loss, polyphagia and nocturia among the two studied groups. Additionally, DKA group had obvious typical symptoms suspecting DKA diagnosis (vomiting, abdominal pain, weakness, disturbed conscious level, respiratory distress and dehydration) (Fig. 1).

Delayed diagnosis (was defined as $>72 \mathrm{~h}$ between the recognition of symptoms and diagnosis of diabetes) was recorded in $98.1 \%$ and $58.7 \%$ of DKA and non DKA groups respectively, and was significantly higher in DKA group $(P=0.014)$. Misdiagnosis was reported in $69.8 \%$ of the DKA group as respiratory problems $(30.2 \%)$, gastroenteritis $(26.4 \%)$ and urinary tract infections (1.9\%). Mismanagement in the form of delayed initiation of insulin therapy after diabetes diagnosis was recorded in 15 patients $(28.3 \%)$ of the DKA group versus only one patient $(2.2 \%)$ in non DKA group with statistically significant difference between 


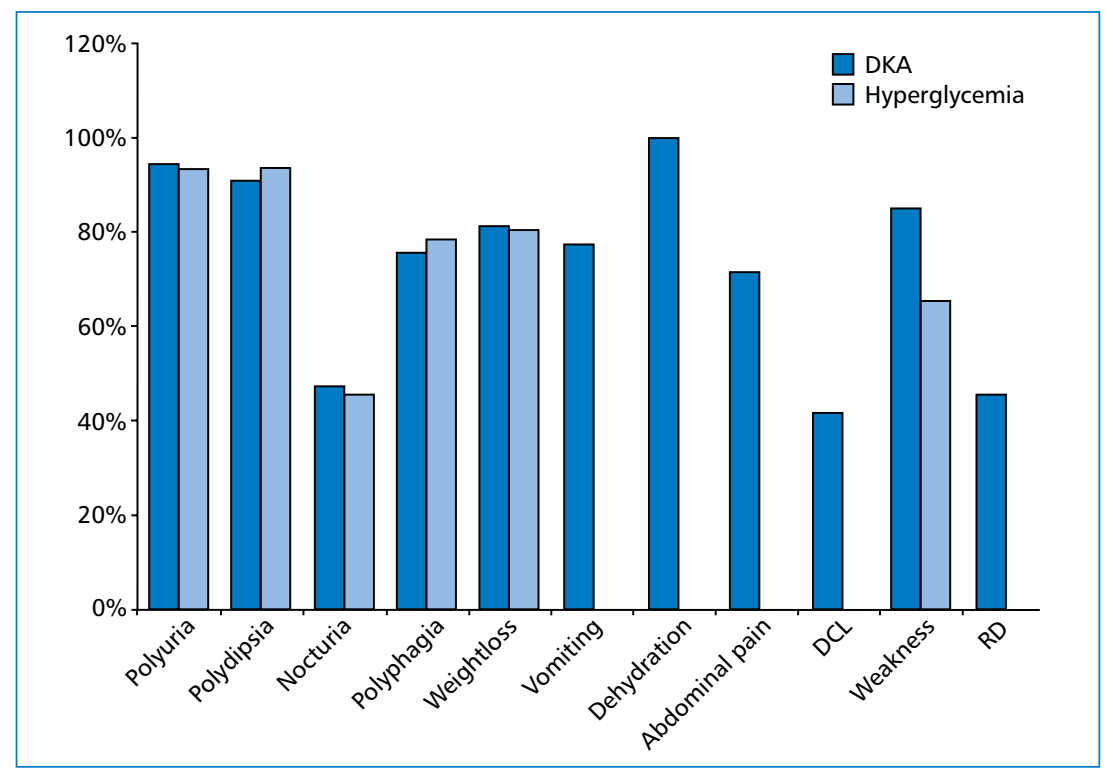

Figure 1. The common presenting symptoms in diabetic ketoacidosis group (DKA) and non DKA group. DCL — disturbed conscious level; RD — respiratory distress

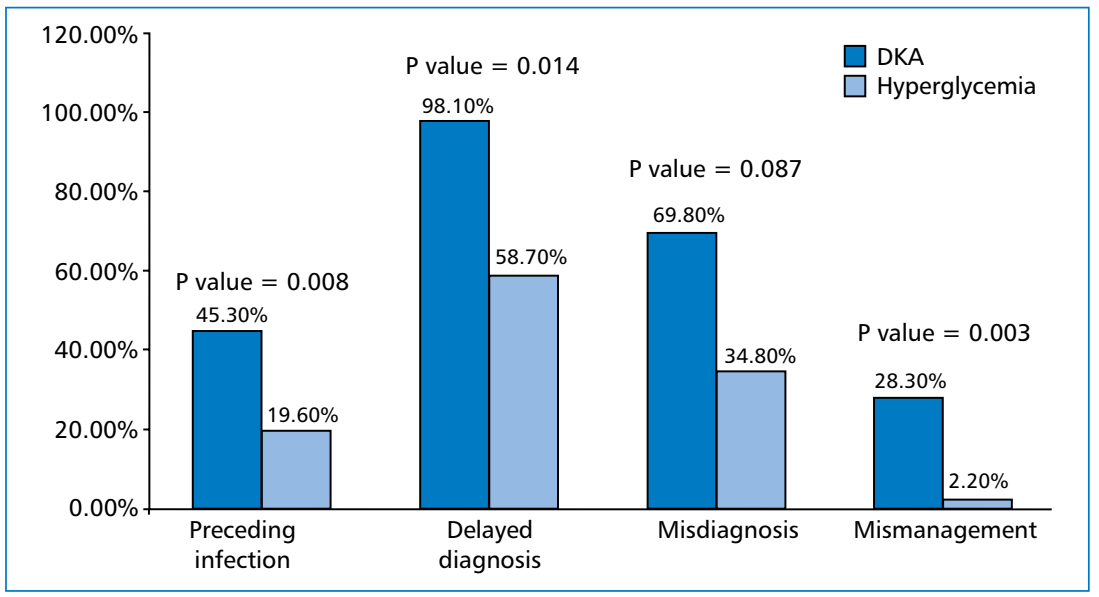

Figure 2. Precipitating factors for DKA development. $P$ value less than 0.05 is significant

the two groups $(P$ value $=0.003)$ (Fig. 2). Forms of mismanagement included prescribing diet modification (amount and type) only, diet modification and exercise, diet modification with abstinence from sugar and/or oral hypoglycemic drugs. Logistic regression analysis to identify the most significant risk factor(s) associated with the development of DKA in children with newly diagnosed type 1 diabetes showed delayed start of insulin therapy as the most significant risk factor with $(\mathrm{OR}=1.267, \mathrm{P}=0.023)$ (Table 2).

\section{Discussion}

The frequency of DKA at the onset of diabetes in children varies considerably from country to country.
This short term prospective study aimed to investigate the frequency and risk factors associated with the development of diabetic ketoacidosis in Egyptian infants and children below 15 years of age with new onset type 1 diabetes. The frequency of DKA at presentation was $53.5 \%$, with nearly half of the cases with severe DKA (severe DKA in 49\%, moderate DKA in $45.3 \%$, and mild DKA in $5.7 \%$ ). The same high frequency was also reported by a previous study [12] from the same center which was $51.5 \%$ among children with newly diagnosed diabetes. Additionally, an earlier study conducted in another tertiary referral center in Egypt reported the frequency of DKA at T1DM diagnosis was $60.57 \%$ [15]. These findings confirms the high frequency and severity 
Table 2. Logistic regression analysis for variables predicting the development of DKA at diagnosis of diabetes

\begin{tabular}{lcccc}
\hline Risk factors for developing DKA & Odds ratio & \multicolumn{2}{c}{ 95\% CL for OR } & P value \\
\cline { 2 - 4 } & & Upper & Lower & \\
\hline Chronological age (years) & 0.962 & 1.043 & 0.887 & 0.343 \\
Time lapse between onset of symptoms and diagnosis of diabetes (hours) & 0.992 & 1.011 & 0.973 & 0.407 \\
Time lapse between diagnosis of diabetes and start of insulin treatment (hours) & 1.267 & 1.552 & 1.034 & 0.023 \\
\hline
\end{tabular}

DKA — diabetic ketoacidosis

of DKA at the time of type 1 diabetes diagnosis among Egyptian children.

The frequency of DKA at the onset of type 1 diabetes from different countries other than Egypt ranged from $12.8 \%$ to $80 \%$ with the highest frequency in Saudi Arabia and United Arab Emirates. However, the lowest incidence was reported in Canada and Sweden [6]. Mbugua et al. [16] suggested the explanation for the different prevalence rates includes differences in study population, the background prevalence of diabetes in the given population, presence or absence of family history of T1DM, as well as the definition of DKA used in the particular study.

In agreement with previous reports $[12,17]$ the most common symptoms preceding the diagnosis were polyuria, polydipsia, weight loss, polyphagia and nocturia among the two studied groups. However, the incidence of infection and febrile illness were significantly higher among the ketoacidosis group. This was in agreement with the results of Xin et al. [18] and Rayfield et al. [19] who also reported that history of infection could increase the danger of development of diabetic ketoacidosis and explained that the cause could be attributed to insulin resistance that result from the release of inflammatory cytokines and the counter regulatory response, also the infection itself could mask the early symptoms of diabetes.

Misdiagnosis was reported in about $70 \%$ of DKA group. Most of the patients were misdiagnosed as respiratory problems or gastroenteritis. Misdiagnosis also can lead to delayed diagnosis.

Delayed diagnosis in the present study was defined as more than $72 \mathrm{~h}$ between recognition of symptoms and diagnosis of diabetes. This was significantly more frequent among the DKA group when compared to the non DKA group. We assume that one of the contributing factors to the delay in diagnosis was the high rate of occurrence of infection at diabetes presentation, which deviated the management to the apparent infection without suspecting diabetes. This confirms the necessity of raising the awareness regarding the presenting clinical features of diabetes in children as well as stressing on the importance of blood glucose assessment in any unwell child.
Delayed diagnosis was also previously reported by Sundaram et al. [20] in the UK who found that more than $\mathbf{2 4}$ hour delay in the diagnosis of diabetes symptoms can increase the diabetic ketoacidosis risk presentation. However, a systematic review conducted by Uscher-Smith et al. [21] found that delayed diagnosis itself as a risk factor for DKA presentation was not consistent among the studies.

Mismanagement with delayed start of insulin therapy after diabetes diagnosis was detected in $28.3 \%$ of the DKA group versus only one patient in non DKA group, with statistically significant difference between the two groups. Mismanagement after being diagnosed with diabetes included one of the following measures: diet modification alone, exercise with diet modification, abstinence from sugar with or without added oral hypoglycemic drugs. Although initiation of insulin therapy immediately after T1DM diagnosis is the mainstay treatment. Refusal of insulin as the primary line of treatment is a major problem among Egyptian families. Insulin is usually perceived as terminal measure in the treatment plan of diabetes. Also there is a misconception almost always expressed by the parents that insulin injection will suppress the endogenous pancreatic secretion and hinder the recovery of pancreatic cells. In addition, the belief that diabetes in children is essentially caused by factors like psychological stress or overconsumption of sweets and sugary food make parents persuading measures of intervention other than the start of insulin injections.

Multivariate analysis to determine the most significant precipitating factor(s) associated with the development of DKA at the time of diabetes diagnosis revealed that delayed initiation of insulin therapy, in spite of diagnosis of diabetes was the most significant risk factor associated with development of DKA (OR $=1.267$, P value $=0.023$ ). Lévy-Marchal et al. [8] reported in a multicentre study across Europe that the delay more than 24 hours between diagnosis and treatment was associated with a small increased risk of developing diabetic ketoacidosis among children (odds ratio 1.74 [1.10 to 2.77]).

Other studied factors, age at onset of diabetes and time lapse between onset of diabetes symptoms and 
diagnosis of diabetes were statistically comparable. Residence area (urban, slum or rural), and parental consanguinity also didn't reach statistically significant difference between the two groups. This is in agreement with other previous studies [21, 22].

Concerning consanguinity, $25.5 \%$ of all patients were offspring of consanguineous parents but there was no significant difference between the two groups regarding parental consanguinity. This finding was concordant to what was reported by Usher-Smith et al. [21] in their systematic review who found that parental consanguinity failed to show a significant difference in the rate of diabetic ketoacidosis in children of consanguineous parents.

The present study also showed that positive family history of diabetes was found in $54.5 \%$ of all patients, $56.6 \%$ of them presented with DKA with significantly higher number of first degree relative with diabetes among this group. The majority of cases had relatives with type 2 diabetes. The present study revealed that the presence of positive family history of diabetes, unfortunately, was not protective and did not prevent progression to DKA by recognizing symptoms at earlier stage. This might indicate low awareness of clinical features and treatment of diabetes in children as well as the refusal of the families with a positive family history of diabetes to accept the reality that children are susceptible to develop diabetes.

Awareness should clearly target the necessity of the immediate initiation of insulin therapy once diabetes is diagnosed in the pediatric age group, and the high risk of ketoacidosis if delayed. This should target health care professionals as well as the community as a whole as apparently acceptance of insulin therapy is commonly rejected and feared by parents, and not always perceived as urgent by health care professionals.

\section{Conclusion}

The prevalence of DKA is high among Egyptian children at diagnosis of type 1 diabetes. It is not only caused by misdiagnosis and mismanagement of diabetes, but also delayed initiation of insulin therapy in those diagnosed. This highlights the importance of increasing awareness concerning clinical features of diabetes in children and the urgency of insulin therapy among primary health care professionals and the community as a whole.

\section{Limitation}

Our study had some limitations, the small sample size and the short period of assessment ( 3 months). Further detailed analysis of patients according to different age groups could not be done due to small sample size. Further larger and multicenter studies are needed to answer that unanswered question whether DKA is a result of an aggressive disease form or it is a result of delayed diagnosis and delayed initiation of insulin therapy.

\section{Conflict of interest}

The authors declare absence of conflict of interest and that they had complete access to data and information relevant to the study.

The study protocol was approved by Research Committee of the Pediatric Department, Faculty of Medicine, Cairo University, on 20/9/2015, approval number not applicable.

\section{REFERENCES}

1. Maahs DM, West NA, Lawrence JM, et al. Epidemiology of type 1 diabetes. Endocrinol Metab Clin North Am. 2010; 39(3): 481-497, doi: 10.1016/j.ecl.2010.05.011, indexed in Pubmed: 20723815.

2. IDF DIABETES ATLAS. Ninth edition 2019.

3. Arab M. Diabetes mellitus in Egypt. World Health Stat Q. 1992; 45(4): 334-337, indexed in Pubmed: 1299073.

4. Salem MA, Tantawy AA, Radwan M, et al. Clinicoepidemiological study of type 1 diabetes mellitus in Egyptian children and adolescents. Pediatr Diabetes . 2010; 11: 94-95.

5. El-Ziny MAM, Salem NAB, El-Hawary AK, et al. Epidemiology of childhood type 1 diabetes mellitus in Nile Delta, northern Egypt - a retrospective study. J Clin Res Pediatr Endocrinol. 2014; 6(1): 9-15, doi: 10.4274/Jcrpe.1171, indexed in Pubmed: 24637304.

6. Usher-Smith JA, Thompson M, Ercole A, et al. Variation between countries in the frequency of diabetic ketoacidosis at first presentation of type 1 diabetes in children: a systematic review. Diabetologia. 2012; 55(11): 2878-2894, doi: 10.1007/s00125012-2690-2, indexed in Pubmed: 22933123

7. Olak-Białoń B, Deja G, Jarosz-Chobot P, et al. Ocena wystepowania i analiza wybranych czynników ryzyka kwasicy ketonowej w momencie ujawnienia cukrzycy typu 1 [The occurrence and analysis of chosen risk factors of DKA among children with new onset of DMT1]. Pediatr Endocrinol Diabetes Metab. 2007; 13: 85-90.

8. Lévy-Marchal C, Patterson CC, Green A, et al. EURODIAB ACE Study Group. Europe and Diabetes. Geographical variation of presentation at diagnosis of type I diabetes in children: the EURODIAB study. European and Dibetes. Diabetologia. 2001; 44 Suppl 3: B75-B80, doi: 10.1007/pl00002958, indexed in Pubmed: 11724421.

9. Dabelea D, Rewers A, Stafford JM, et al. SEARCH for Diabetes in Youth Study Group. Trends in the prevalence of ketoacidosis at diabetes diagnosis: the SEARCH for diabetes in youth study. Pediatrics. 2014; 133(4): e938-e945, doi: 10.1542/peds.20132795, indexed in Pubmed: 24685959.

10. Elaziz DS, Hafez MH, Galal NM, et al. CD4(+) CD25(+) cells in type 1 diabetic patients with other autoimmune manifestations. J Adv Res. 2014; 5(6): 647-655, doi: 10.1016/j.jare.2013.09.004, indexed in Pubmed: 25685533.

11. Samahy MH, Elbarbary NS, Elmorsi HM. Current status of diabetes management, glycemic control and complications in children and adolescents with diabetes in Egypt. Where do we stand now? And where do we go from here? Diabetes Res Clin Pract. 2015; 107(3): 370-376, doi: 10.1016/j.diabres.2015.01.004, indexed in Pubmed: 25649911.

12. Hassan M, Hafez M, Koura $H$, et al. Etiology, risk factors, clinical features, and pitfalls in the management of newly diagnosed diabetic children and adolescents. Medical Research Journal. 2015; 14(2): 59-65, doi: 10.1097/01.mjx.0000472994.55365.41. 
13. Hassan MM, Arafa N, Abdou M, et al. Characteristics of diabetes diagnosis and control in toddlers and preschoolers from families with limited resources: A single center experience. Diabetes Res Clin Pract. 2020; 159: 107966, doi: 10.1016/j.diabres.2019.107966, indexed in Pubmed: 31805353.

14. Mayer-Davis EJ, Kahkoska AR, Jefferies C, et al. ISPAD Clinical Practice Consensus Guidelines 2018: Definition, epidemiology, and classification of diabetes in children and adolescents. Pediatr Diabetes. 2018; 19 Suppl 27: 7-19, doi: 10.1111/pedi.12773, indexed in Pubmed: 30226024.

15. Samahy MH, Elbarbary NS, Elmorsi HM, et al. Epidemiology and management of type 1 diabetes mellitus at the $a$ in shams university pediatric hospital. J Egypt Public Health Assoc. 2008; 83(1-2): 107-132, indexed in Pubmed: 18992206.

16. Otieno CF, Kayima JK, Mbugua PK, et al. Diabetic ketoacidosis: clinical presentation and precipitating factors at Kenyatta $\mathrm{Na}$ tional Hospital, Nairobi. East Afr Med J. 2005; 82(12 Suppl): S191-S196, doi: 10.4314/eamj.v82i12.9381, indexed in Pubmed: 16619732.

17. Habib HS. Frequency and clinical characteristics of ketoacidosis at onset of childhood type 1 diabetes mellitus in Northwest
Saudi Arabia. Saudi Med J. 2005; 26(12): 1936-1939, indexed in Pubmed: 16380776.

18. Xin $Y$, Yang $M$, Chen $X J$, et al. Clinical features at the onset of childhood type 1 diabetes mellitus in Shenyang, China. J Paediatr Child Health. 2010; 46(4): 171-175, doi: 10.1111/j.14401754.2009.01657.x indexed in Pubmed: 20546479.

19. Rayfield E, Ault M, Keusch G, et al. Infection and diabetes: The case for glucose control. The American Journal of Medicine. 1982; 72(3): 439-450, doi: 10.1016/0002-9343(82)90511-3.

20. Sundaram PCB, Day E, Kirk JMW. Delayed diagnosis in type 1 diabetes mellitus. Arch Dis Child. 2009; 94(2): 151-152, doi: 10.1136/ adc.2007.133405, indexed in Pubmed: 18562452.

21. Usher-Smith JA, Thompson MJ, Sharp SJ, et al. Factors associated with the presence of diabetic ketoacidosis at diagnosis of diabetes in children and young adults: a systematic review. BMJ. 2011; 343: d4092, doi: 10.1136/bmj.d4092, indexed in Pubmed: 21737470.

22. Sadauskaite-Kuehne V, Samuelsson U, Jasinskiene E, et al. DEBS Study Group. Severity at onset of childhood type 1 diabetes in countries with high and low incidence of the condition. Diabetes Res Clin Pract. 2002; 55(3): 247-254, doi: 10.1016/s01688227(01)00328-x, indexed in Pubmed: 11850101. 